\title{
A somatic mutation in PIK3CD unravels a novel candidate gene for lymphatic malformation
}

\author{
Shengcai Wang ${ }^{1 \dagger}$, Wei Wang ${ }^{2 \dagger}$, Xuexi Zhang ${ }^{1 \dagger}$, Jingang Gui ${ }^{2}$, Jie Zhang ${ }^{1}$, Yongli Guo ${ }^{3}$, Yuanhu Liu', Lin Han ${ }^{4}$, \\ Qiaoyin Liu', Yanzhen $\mathrm{Li}^{1}$, Nian Sun ${ }^{1}$, Zhiyong Liu' ${ }^{1}$, Jiangnan Du${ }^{1}$, Jun Tai ${ }^{1 *}$ and Xin $\mathrm{Ni}^{i^{*}}$
}

\begin{abstract}
Background: Lymphatic malformations (LMs) are benign congenital malformations that stem from the abnormal development of the lymphatic vessels during early embryogenesis. Somatic PIK3CA gene mutations are conventional cause leading to LMs. Both macrocystic and microcystic LMs arise due to lymphatic endothelial cell-autonomous defects, depending on the time in development at which PIK3CA gene mutation occurs. Recent study finds a PIK3CA mutation in $79 \%$ of LMs. However, discovering new genetic events in this disease is crucial to identify the molecular mechanism of the pathogenesis and further develop new targeted therapies.
\end{abstract}

Results: Here, we initially performed whole-exome sequencing in six children with LMs to find a new causal gene. Somatic mutations in PIK3CA (c.1633G > A [p. E545K] and PIK3CD (c.1997T > C [p.L666P]) were discovered in two different individuals. In vitro functional studies were conducted to demonstrate the pathogenicity of the novel mutation c.1997T > C in PIK3CD. We found that L666P promoted the cell proliferation and migration of human umbilical vein endothelial cells (HUVECs) and induced hyperactivation of the mTOR pathway. These findings indicate that the PIK3CD mutation affects downstream signalling in endothelial cells, which may impair normal lymphangiogenesis.

Conclusions: This study reveals a novel candidate gene associated with the development of LMs, which is consistent with previous researches. These findings in our study may offer a novel gene target for developing therapies, which acts in tight interaction with the previously known PIK3CA.

Keywords: Lymphatic malformations, Whole-exome sequencing, PIK3CD, Novel mutations, MTOR pathway

\section{Introduction}

Lymphatic malformations (LMs) are congenital lesions caused by defects in the development of the lymphatic system and mainly observed in neonates or young children, with an incidence of 1 in 4000 to 1 in 2000 [1]. Lymphatic malformations (LM) are characterized by the

\footnotetext{
*Correspondence: trenttj@163.com; nixin@bch.com.cn

tJoint first authors who contributed equally to this work.

${ }^{1}$ Department of Otolaryngology-Head and Neck Surgery, Beijing Children's Hospital, Capital Medical University, National Center for Children's Health, Beijing 100045, China

Full list of author information is available at the end of the article
}

overgrowth of lymphatic vessels during pre- and postnatal development [2]. LMs can cause adjacent structures compromise leading to airway obstruction even dyspnea, cosmetic deformity, swallowing impairment, infection or naturally diffuse, especially in head and neck region. Conservative observation and surgery are main treatments and the latter including surgical excision (e.g., partial or total excision), sclerotherapy, radiofrequency ablation, laser therapy. With the development of medical genetics of LMs, new therapies increasingly emerged such as oral medications (i.e., sildenafil, propranolol, and sirolimus), and vascularized lymph node transfer [3, 4]. A systematic review recruited 20 trials including 71 patients with oral otherwise in a credit line to the material. If material is not included in the article's Creative Commons licence and your intended use is not permitted by statutory regulation or exceeds the permitted use, you will need to obtain permission directly from the copyright holder. To view a copy of this licence, visit http://creativecommons.org/licenses/by/4.0/. The Creative Commons Public Domain Dedication waiver (http://creativecommons.org/publicdomain/zero/1.0/) applies to the data made available in this article, unless otherwise stated in a credit line to the data. 


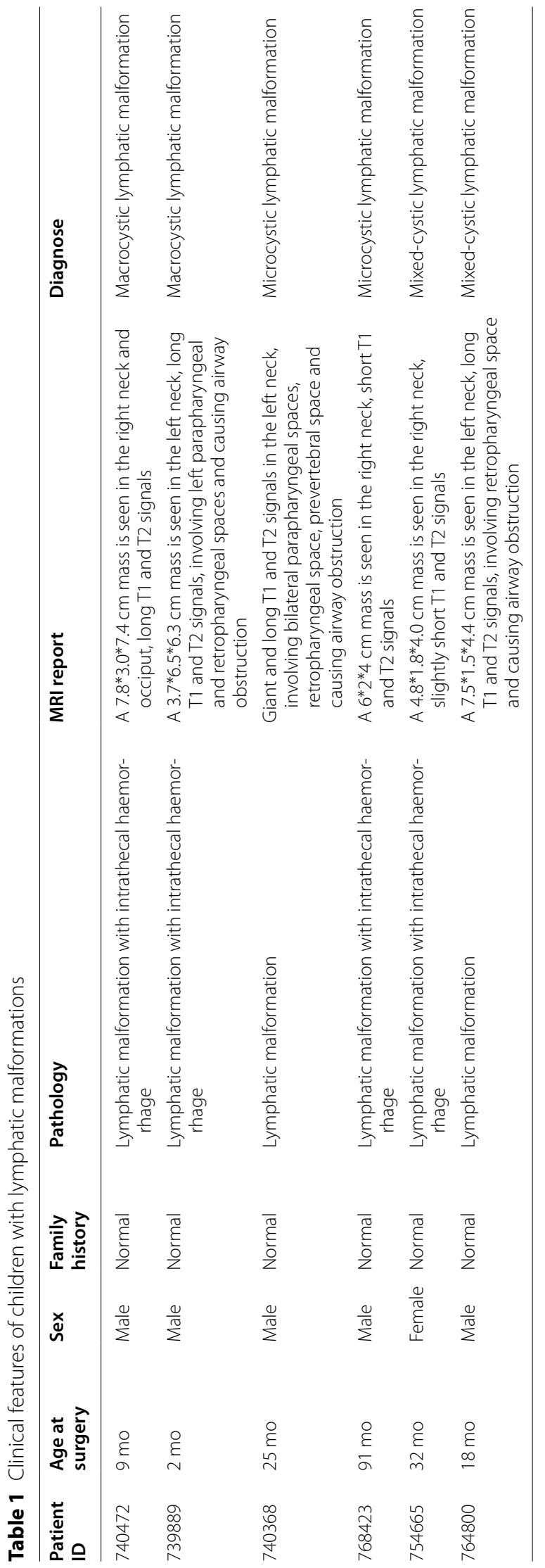


sirolimus, and found that the sirolimus was effective for LMs [4]. However, patients with extensively infiltrating LMs, namely, intractable LMs (iLM), experienced high risks of recurrence and progression, routine treatment regimens are less effective for iLMs [5].

Recently, increasing evidence shows that the PI3K/ $\mathrm{AKT} / \mathrm{mTOR}$ pathway is involved in the pathogenesis of isolated LMs and syndromic disorders in which LM is a component feature [6-9]. For example, proteus syndrome patients who also have LMs carried a somatic mutation in $A K T 1$, which encodes RAC-alpha serinethreonine protein kinase and plays a role in lymphangiogenesis $[9,10]$. Moreover, somatic mutations that activate phosphatidylinositol 4,5-bisphosphate 3-kinase catalytic subunit alpha (PIK3CA) have been found in approximately $79 \%$ of LMs $[8,11]$. Thus around $20 \%$ remain unexplained. Somatic PIK3CA mutation is not only identified in isolated LM, but also in CLOVES syndrome or Klippel-Trenaunay-Weber syndrome [9]. High activity of the PI3K-AKT- mTOR pathway was demonstrated by hyperphosphorylation of AKT-Ser473 in all LM-derived lymphatic endothelial cells (LECs) as compared to normal LECs while LM-derived fibroblasts did not possess such mutations [12]. Several PIK3CA somatic mutations have been shown to be oncogenic as well as function as the main pathogenic mechanisms of LMs and vascular malformations by promoting the hyperproliferation of endothelial cells [13]. The International Society for the Study of Vascular Anomalies (ISSVA) has also identified a PIK3CA mutation as a specific pathogenic cause for LMs. However, the molecular mechanism of LMs without PIK3CA mutations is still unclear, and other genetic alterations have not been found in the disease. Thus, the discovery of new genetic events in LMs is crucial to identify the molecular mechanism of the pathogenesis and further develop novel targeted therapies. In this study, a novel candidate mutation in PIK3CD was identified as an LM-associated mutation by whole-exome sequencing (WES) and was validated by in vitro functional studies.

\section{Results}

\section{Diagnosis and characteristics of LMs}

Preoperative diagnoses were based on the clinical and radiological findings (Table 1 ). The maximum diameter of every single cyst was measured by magnetic resonance imaging (MRI). Based on the size of the cyst, the LMs were morphologically classified as macrocystic LMs (the smallest cyst more than $2 \mathrm{~cm}$ in macrocystic malformations), microcystic LMs (the biggest cyst less than $2 \mathrm{~cm}$ in microcystic malformations) or mixed cystic LMs (containing both macrocystic and microcystic proportion of malformations). Axial T2 MRI demonstrated that most of the cervical LMs with high signal intensity comprised fluid component and septations, while heterogeneous areas of hypointensity usually suggested a haemorrhage. The extensive lesions caused airway obstruction, involving the trachea, blood vessels, parapharyngeal spaces, retropharyngeal space and prevertebral space (Fig. 1). Macrocystic LMs (ID: 740472, 739889) were composed of vessels with a single layer of flattened epithelial cells, including smooth muscle cells, with variable thickness. Microcystic LMs (ID: 740368,768423$)$ were irregularly shaped lesions resulting from the localized collection of abnormal and cystically dilated lymph vessels filled with lymph fluid. Mixed cystic LMs (ID: 754665, 764800) contained morphology representative of a mixture of micro- and macrocystic LMs (Additional file 1: Figure S1).

\section{Mutations in PIK3CA and PIK3CD were identified in children with LMs}

Peripheral blood samples and lymphatic tissue specimens from 6 patients were collected for WES and digital polymerase chain reaction (PCR) verification. A total of 16 variants associated with mTOR pathway were identified in 6 genes, including IRS1, MTOR, PIK3CA, PIK3CD, TSC1 and TSC2. For other candidate variants, no mutant droplet is detected (mTOR and TSC2) or ddPCR failed (TSC1 and IRS1, primers failed to distinguish wt and mut sequences). Finally, only two variants, c.1633G >A (p. E545K) in PIK3CA (NM_006218) and c.1997T >C (p.L666P) in PIK3CD (NM_005026), were identified in 2 patients (Table 2 and Fig. 2). The mutation frequency of PIK3CA was $6.06 \%$ $(4 / 66)$ as detected by WES and $2.14 \%(6 / 281)$ by digital PCR, and that of PIK3CD was $1.73 \%(5 / 289)$ and $2.81 \%$ $(2 / 71)$, respectively. We analysed the pathogenicity of the mutations mainly based on criteria from the Association for Molecular Pathology (AMP) Clinical Practice guidelines [14]. Mutation of c.1633G $>\mathrm{A}$ in PIK3CA has been reported as a disease-causing mutation associated with lymphatic disorders. The novel mutation c.1997T > C in PIK3CD has never been reported before in public databases for somatic mutations (COSMIC). Functional predictions by multiple software programs (MutationTaster, Provean and PolyPhen-2) demonstrated that these mutations are damaging and deleterious. The site of PIK3CD mutation, lysine 666, is highly conserved across many species and is located in the PIK helical domain (Fig. 3). Both mutations are associated with the PI3K/AKT/mTOR pathway; thus, we considered them variants with potential clinical significance. It should be noted that somatic activating mutations in 

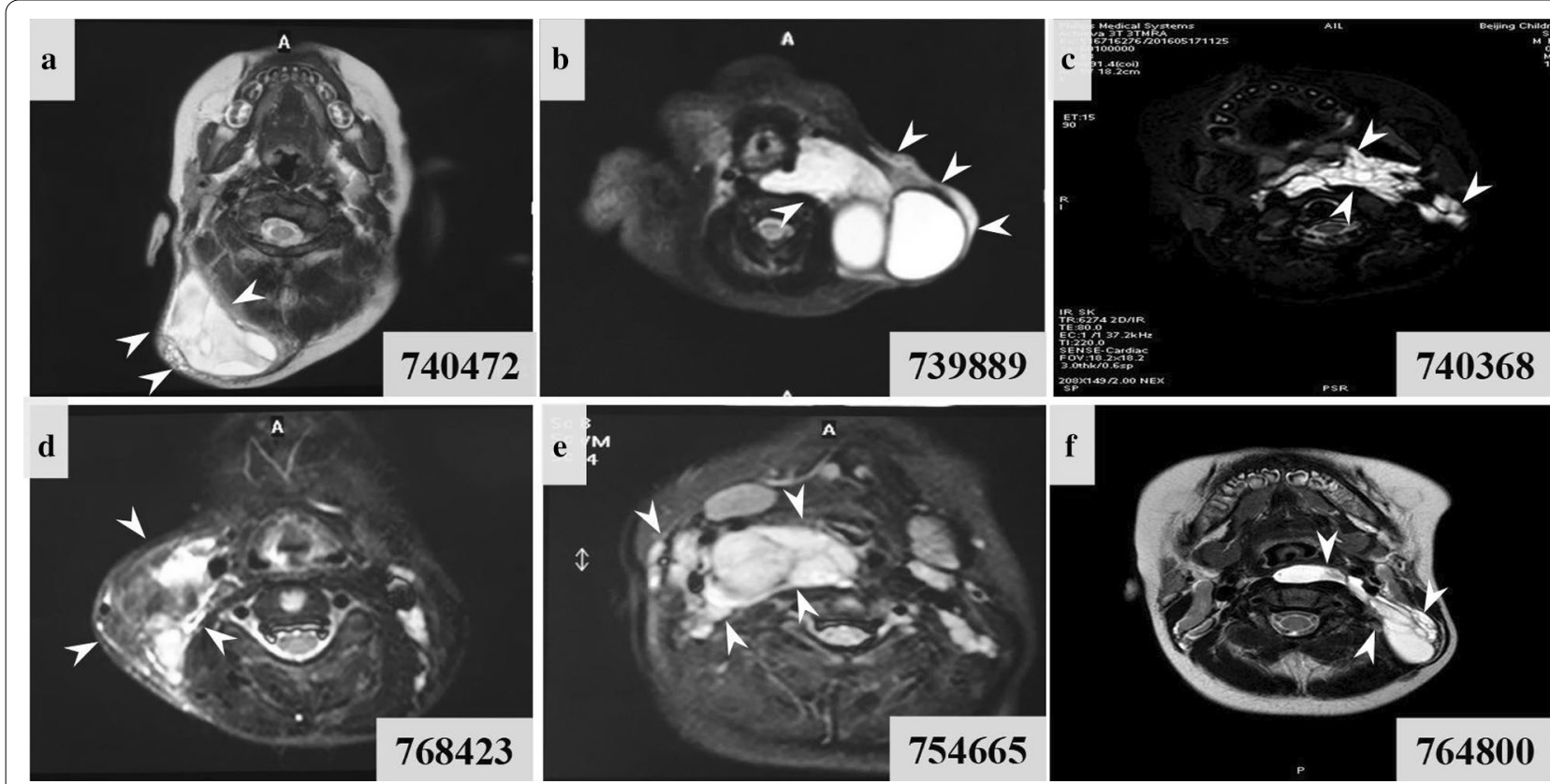

Fig. 1 MRI image of each subtype of LMs. a, b MRI (T2 image in axial view) reveal macro-cystic lesions (more than $2 \mathrm{~cm}$ in diameter) in head and neck region. c MRI (T2 image in axial view) reveals micro-cystic lesion in parapharyngeal spaces, retropharyngeal space, and prevertebral space. $\mathbf{d}$ The axial postgadolinium contrast enhanced T1-weighted MRI LM with microcysts, with enhancing setae between the individual cysts. e, f MRI (T2 image in axial view) reveal macrocysts and microcysts

the PIK3CA gene have been detected in LMs, but this is the first report of the PIK3CD mutation in this disease.

\section{The PIK3CD mutation promoted cell proliferation} and migration in human umbilical vein endothelial cells (HUVECs)

We performed in vitro functional studies to demonstrate the pathogenicity of the identified PIK3CD mutation and how PIK3CD affects the movement of endothelial cells. HUVECs overexpressing wild-type and mutant PIK3CD (WT- and MT-HUVEC, respectively) via adenovirus infection were created, with empty vector (Ctrl-HUVEC) serving as a control (Fig. 4a). The infection efficiencies were validated as greater than $90 \%$ by GFP fluorescence imaging (Fig. $4 \mathrm{~b}$ ). The CCK8 assay was conducted to determine their proliferation capability. The WT-HUVECs presented a similar proliferation rate as the control group, but it appeared to increase significantly at the $72 \mathrm{~h}$ time point (Fig. 4c, d). The results of scratch assay showed fastest wound closure in MT-HUVEC (PIK3CD-expressing) compared with vehicle treated (Ctrl) primary HUVEC and wild type at $12 \mathrm{~h}$ post scratching. (Fig. 4e, f). These results suggested that exogenous overexpression of mutant PIK3CD increased the proliferative and migration capabilities of HUVECs.

\section{The PIK3CD mutation induced the hyperactivation of the mTOR pathway}

We next analysed the activation status of AKT, mTOR and S6, which are critical downstream targets of PI3K (Fig. 5a). Real-time qPCR revealed that the mRNA expression levels of these downstream targets were significantly increased and most pronounced in the MT-HUVECs, followed by the levels in the WT- and Ctrl-HUVECs (Fig. 5b). Furthermore, the protein levels of phosphorylated AKT, mTOR and S6 were strongly upregulated in MT-HUVECs compared with WT- and Ctrl-HUVECs. (Fig. 5c, d). At both the mRNA and protein level, mTOR expression was not affected by the overexpression of wild-type PIK3CD. The results above indicated the significant hyperproliferative phenotypes in MT-HUVECs and the slight increase in proliferation of WT-HUVECs compared with that of the 


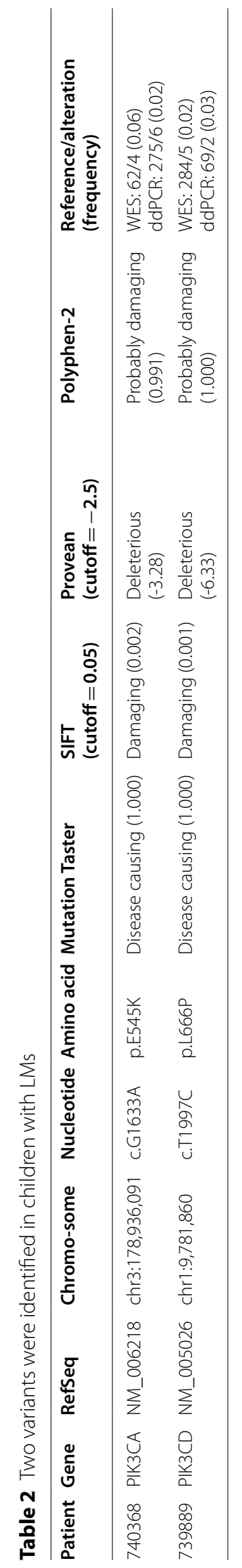




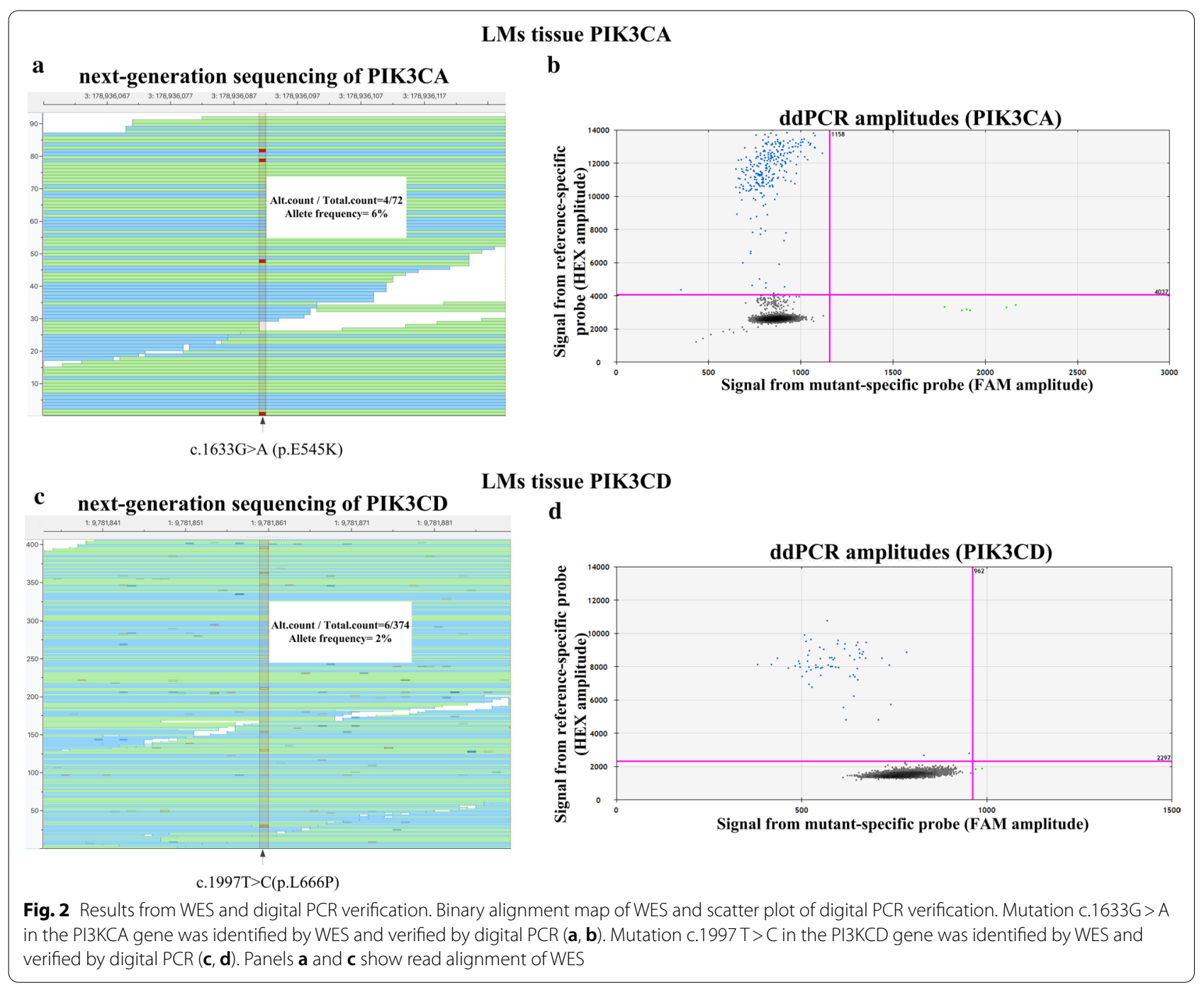

Ctrl-HUVECs. They also supported our hypothesis that this PIK3CD mutation is pathogenic.

\section{Discussion}

PI3Ks are a family of lipid kinases with critical roles in cell biology, including cell proliferation, differentiation, migration and survival [15-17]. There are three categories of PI3Ks (Class IA, Class IB; Class II; and Class III). Class IA PI3Ks comprise a p110 catalytic subunit and a $\mathrm{p} 85$ regulatory subunit. The $\mathrm{p} 110 \alpha, \mathrm{p} 110 \beta$ and $\mathrm{p} 110 \delta$ catalytic isoforms are encoded by the PIK3CA, PIK3CB and $P I K 3 C D$ genes, respectively. $\mathrm{p} 110 \alpha$ is frequently involved in human cancers, including endometrial, breast, ovarian, colorectal and other various tumours, by affecting cell proliferation, migration and survival [18-22]. Most of the LMs were caused by somatic mutations in the $P I K 3 C A$ gene, which could lead to the hyperproliferation of lymphatic endothelial cells $[7,8,13]$.
Among three Class IA PI3K catalytic isoforms, p110 $\alpha$ and $p 110 \beta$ are ubiquitously expressed, whereas p110 $\delta$ is principally enriched in leukocytes and regulates immune functions [23]. However, some non-leucocytes such as neurons [24], ECs (endothelial cells) [25] and lung fibroblasts [26] also express p1108, albeit at lower levels than in leucocytes. In addition, p110 $\delta$ is generally overexpressed to induce cancer cell growth and invasion by activating the AKT-mTOR pathway in hepatocellular carcinoma, glioma, glioblastoma, neuroblastoma, colorectal cancer and breast cancer [27-30]. It is well known that LMs present some similar characteristics as tumours, such as uncontrolled cell proliferation and extension into surrounding tissues. Lymphatic vascular endothelial cells in LMs usually exhibit abnormal proliferation due to mTOR activation [31]. However, it was unclear whether genetic changes in PIK3CD play a role in the pathogenesis of LMs. All these signs indicated the endothelial 


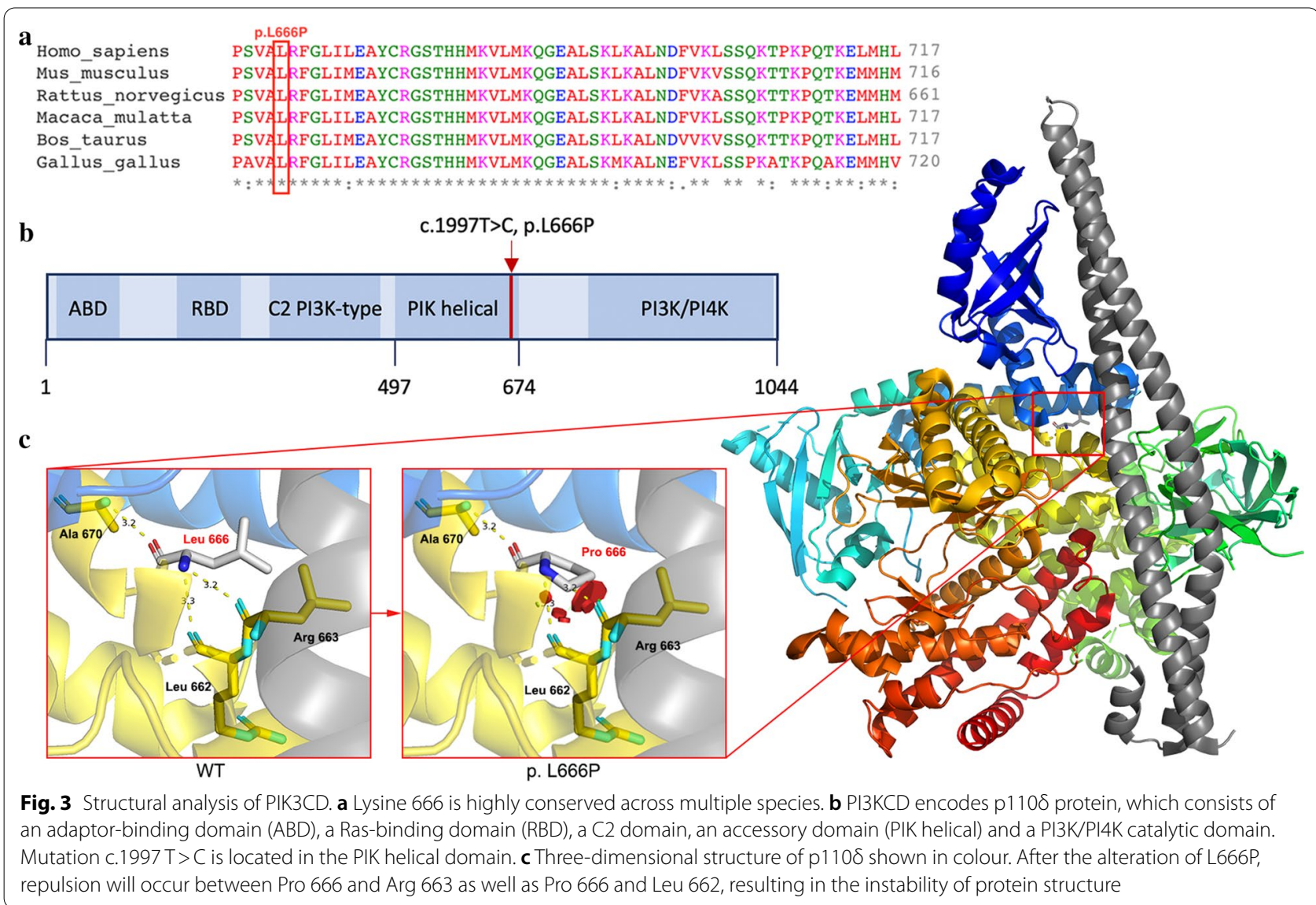

cells overproliferated and aggregated, leading to LMs. In the present study, in vitro functional studies demonstrated that exogenous overexpression of wild-type and most significantly mutant PIK3CD increased the proliferation rate of HUVECs. In addition, phosphorylated protein levels of AKT, mTOR and S6 were significantly increased in cells with exogenous overexpression of the PIK3CD mutant, suggesting that the elevated expression of mutant PIK3CD in vascular endothelial cells may promote the overgrowth of endothelial cells and further affect lymphatic vessel development.

To date, LMs are often treated with rapamycin or rapamycin analogues such as everolimus to cure the lesions and improve quality of life [32]. Accepted paper from our laboratory also demonstrated that rapamycin could effectively reduce volume of LMs especially for Macrocystic LMs [33]. Newly paper showed that a combination of VEGFC inhibition with rapamycin is much more potent inducing even LM regression in mice, although this is a contraindication for VEGF inhibition in children [34].

However, given almost $80 \%$ LM patients carried PI3KCA mutations, mutation-specific inhibitors or combination of inhibitors have become a promising choice for the treatment of LMs. Similar to developmental tumours, LMs carrying a single mutation might be more sensitive to targeted therapies than tumours carrying multiple mutations [35]. Studies have shown that $\mathrm{p} 110 \alpha$-specific inhibitors could normalize aberrant PI3K signalling, thereby reducing or eliminating PIK3CA-driven vascular malformations. The p110 $\alpha$-specific inhibitor BYL719 was also successfully applied for the treatment of patients with PIK3CA-related overgrowth syndrome, which gives hope to patients with LMs [36]. In the future, we will enrol a larger group of patients with LMs to detect the mutation frequency of PIK3CD and to elucidate the mechanism of its pathogenicity. As this is a promising gene for novel targeted therapies, we will also evaluate the effects of PIK3CD mutant-specific inhibitors on the reversal of cellular dysfunction.

\section{Conclusion}

In the present study, we identified a novel PIK3CD somatic mutation in LM, which could serve as a new candidate pathogenic mutation and is presumably involved in the pathogenesis of LMs. In vitro functional studies demonstrated that exogenous overexpression of mutant 


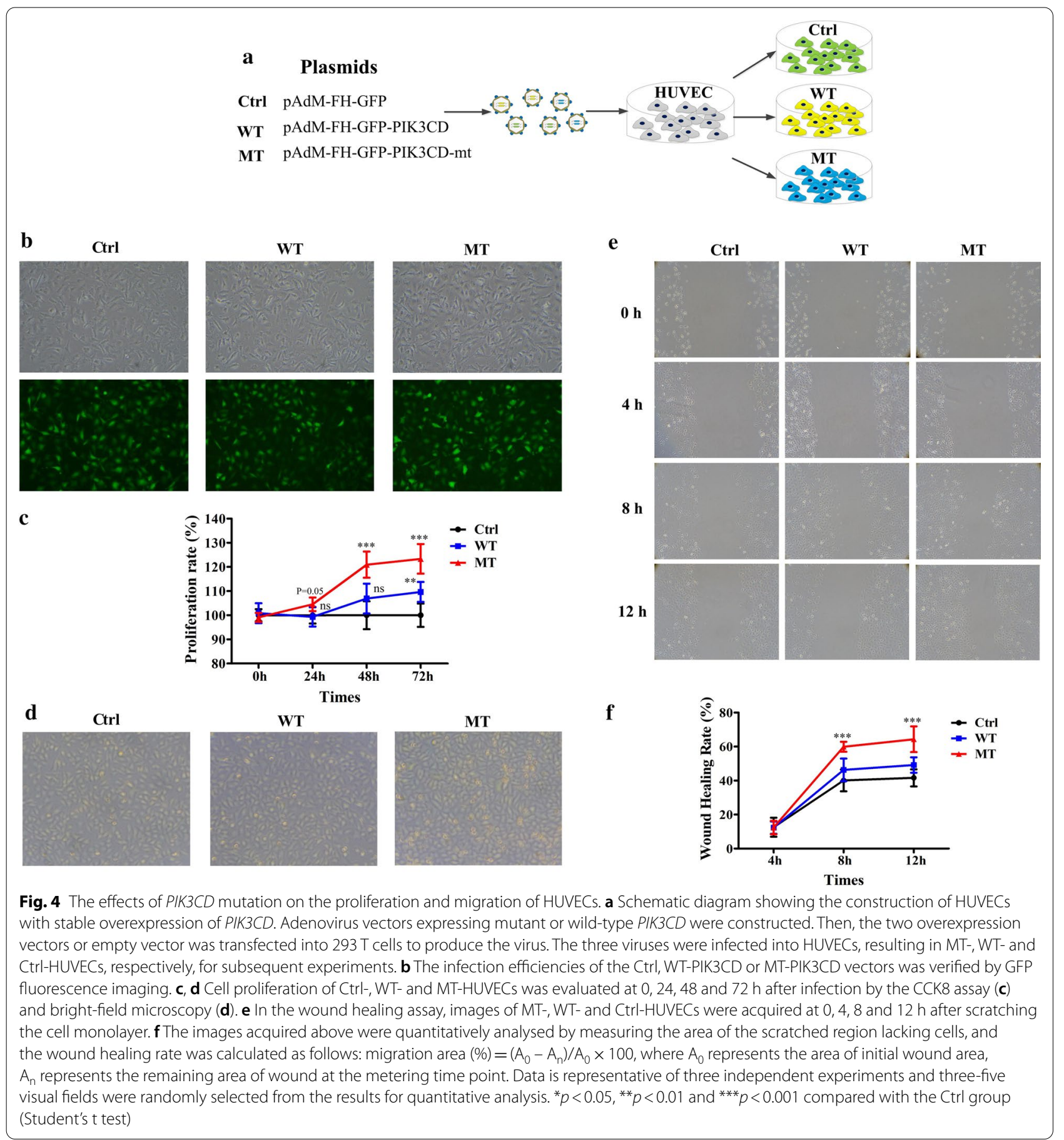

PIK3CD promoted HUVEC proliferation and migration by activating the mTOR pathway. Therefore, PIK3CDinduced cell proliferation of lymphatic vascular endothelial cells and hyperactivation of mTOR signalling might contribute to the pathogenesis of LMs.

\section{Materials and methods}

Patients and sample collection

All 6 patients were admitted to and diagnosed by clinicians in the Department of Otolaryngology at Beijing Children's Hospital Affiliated to Capital Medical University. The clinical characteristics were collected from 

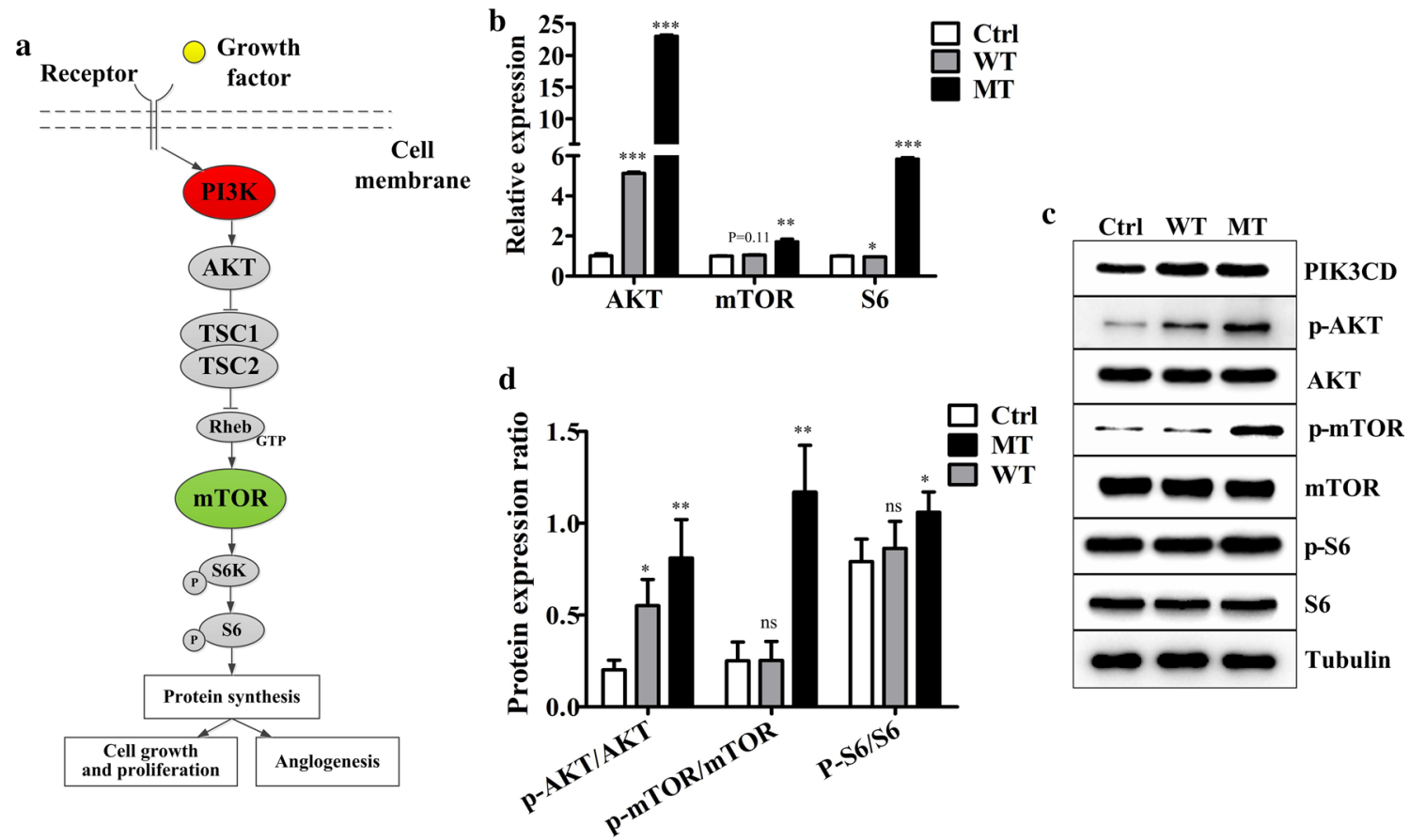

Fig. 5 The identified PIK3CD mutation leads to aberrant activation of the mTOR pathway. a Schematic figure showing that activation of mTOR is regulated by the upstream proteins PIK3 and AKT. b RT-qPCR and $\mathbf{c}$ immunoblot analysis of AKT, mTOR and S6 mRNA and protein expression, respectively, in Ctrl-, WT- and MT-HUVECs. Ctrl, empty vector; WT, wild-type; MT, PIK3CD mutant. d Quantification of the protein band intensity. There are no significant differences between WT and Mut group in p-AKT/AKT level $(p>0.05)$. There are significant differences between WT and Mut group in $p-m T O R / m T O R(p<0.01)$. There are no significant differences between WT and Mut group in $p-S 6 / S 6$ level $(p>0.05)$. Data are presented as the mean $\pm S D$ ( $n=3-5$ per group). ${ }^{*} p<0.05,{ }^{* *} p<0.01$ and ${ }^{* * *} p<0.001$ compared with the Ctrl group (Student's $t$ test)

their medical records (Table 1). Guardians of all the participants signed informed consent forms (ICFs) designed in accordance with the Declaration of Helsinki. The tissue specimens of LMs were obtained under the human subject protocol approved by the Human Ethics Committee of Beijing Children's Hospital Affiliated to Capital Medical University (ID: 2019-k-66, approved on February 2019).

\section{Whole-exome sequencing (WES)}

Peripheral blood and tissue specimens of LMs from all 6 children were sent to Running Gene Inc. (Beijing, China) for WES (Additional file 1: Figure S2). Average depth of coverage was $\times 142$ in blood and $\times 166$ in tissue (Additional file 1: Table S1). DNA samples were isolated from the peripheral blood and lymphatic tissue specimens with a DNA Isolation Kit (Bioteke, AU1802 and AU18016). The DNA concentrations were measured with a Qubit dsDNA HS Assay Kit (Invitrogen, Q32851) on a Qubit fluorometer (Invitrogen, Q33216). High-quality DNA samples were fragmented into 200300 bp by a Covaris Acoustic System (Covaris, Massachusetts, USA), and the resulting DNA fragments were processed with a KAPA Library Preparation Kit (Kapa
Biosystems, KR0453) to construct a DNA library. The libraries were estimated with a Qubit dsDNA HS Assay kit (Invitrogen, Q32851), after which hybridization of pooled libraries to the capture probes was conducted with an Agilent SureSelectXT2 Target Enrichment System (Agilent, Santa Clara, USA). Probe-captured DNA fragments were then enriched by post-capture PCR. The final products were sequenced on an Illumina HiSeq X10 platform (Illumina, San Diego, USA) as 150 bp paired-end reads.

Raw data from the HiSeq X10 platform were processed for quality control and then aligned against the human reference genome (GRCh37/hg19) using the Burrows-Wheeler Alignment tool (http://bio-bwa. sourceforge.net/). Duplicate reads were identified using GATK software (www.broadinstitute.org/gatk), and single-nucleotide polymorphisms and insertions and deletions were examined. Low-quality variants were filtered out based on quality by depth $(<2.0)$, mapping quality $(<40.0)$, Fisher strand $(>60.0)$, mapping quality rank sum test $(<-12.5)$ and read position rank sum test $(<-8.0)$. All the called variants were annotated by ANNOVAR (annovar.openbioinformatics.org/en/latest/) based on public databases (1000 Genomes Project, 
ExAC, gnomAD, ESP6500, CCDS, RefSeq, Ensembl, etc.). The potential impacts of candidate single-nucleotide variants were predicted by the MutationTaster, SIFT, Provean and Polyphen-2 programs.

\section{Germline mutations involved in either PI3K/AKT/mTOR or Ras pathways}

Low-quality variants were filtered out based on quality by depth $(<8.0)$. The remaining variants were filtered against 1000 Genomes Project_EAS, ExAC and gnomAD, with a minor allele frequency $(\mathrm{MAF})<1 \%$ for autosomal and $\mathrm{X}$-linked recessive mutations and an $\mathrm{MAF}<0.01 \%$ for autosomal and X-linked dominant mutations. Based on the Human Gene Mutation Database, nonsense, frameshift, and splicing mutations annotated as disease mutations were retained. Only candidate genes associated with both PI3K/AKT/mTOR and Ras pathways were included. No definite pathogenic germline variant was identified.

\section{Somatic mutations involved in either $\mathrm{PI3K} / \mathrm{AKT} / \mathrm{mTOR}$ or Ras pathways}

Germline mutations appearing in the peripheral blood were filtered out. The remaining mutations were selected based on quality by depth $(<8.0)$ and against 1000 Genomes Project_EAS, ExAC and gnomAD, with an $\mathrm{MAF}<0.01 \%$. Only exonic and splicing variants were included. Synonymous variants and variants with low number of alteration (alt $<4$ ) were excluded as well. Finally, only candidate genes associated with the both $\mathrm{PI} 3 \mathrm{~K} / \mathrm{AKT} / \mathrm{mTOR}$ and Ras pathways were included.

\section{Digital polymerase chain reaction (PCR)}

Digital PCR were conducted to verify the remaining IRS1, MTOR, TSC1, TSC2, PIK3CA and PIK3CD variants. DNA samples were mixed with $2 \mathrm{X}$ ddPCR Supermix for probes (Bio-Rad Laboratories, Inc., USA), probes, primers and $\mathrm{ddH} 2 \mathrm{O}$ (Table 3). The mixture and droplet

Table 3 Sequence of primers and probes used for digital polymerase chain reaction (ddPCR)

\begin{tabular}{ll}
\hline Primer and probes & Sequence $\left(\mathbf{5}^{\prime} \mathbf{-} \mathbf{3}^{\prime}\right)$ \\
\hline PIK3CA-F & GCTCAAAGCAATTTCTACACGA \\
PIK3CA-R & CTTACCTGTGACTCCATAGAAAATC \\
PIK3CA-P-G & 6-FAM-TGAAATCACTGAGCAGGA- BHQ-X \\
PIK3CA-P-A & HEX-TGAAATCACTAAGCAGG-BHQ-X \\
PIK3CD-F & TCCGAGATGCACGTGCC \\
PIK3CD-R & CCTTCATGTGGTGGGTCT \\
PIK3CD-P-T & 6-FAM -TTCGGCCTCATCCT- BHQ-X \\
PIK3CD-P-C & HEX-TTCGGCCCCATCC-BHQ-X \\
\hline
\end{tabular}

generation oil (Bio-Rad Laboratories, Inc., USA) were separately loaded on the DG8 cartridge. Then, targeted droplets were generated by a QX200 Droplet Digital PCR system (Bio-Rad Laboratories, Inc., USA) and transferred to 96-well plates. After PCR in a Bio-Rad thermal cycler T100, the digital PCR data were read and briefly analysed on the QX200 Droplet Digital PCR system.

\section{Cell culture and infection}

Human umbilical vein endothelial cells (HUVECs) were donated by Beijing Belife Bio-Medical Technology LTD and cultured in endothelial cell medium (ECM) (cat no. 1001; ScienCell, San Diego, California, USA) supplemented with $5 \%$ foetal bovine serum (FBS) (cat no. 0025; ScienCell, San Diego, California, USA), 1\% Endothelial Cell Growth Supplement (ECGS) (cat no. 1052; ScienCell, San Diego, California, USA) and 1\% penicillin/streptomycin solution (cat no. 0503; ScienCell, San Diego, California, USA). Cells were cultured at $37{ }^{\circ} \mathrm{C}$ in a humidified atmosphere containing $5 \% \mathrm{CO}_{2}$.

cDNAs coding wild-type PIK3CD (GenBank accession NM_005026.4) or PIK3CD mutations were synthesized for adenovirus packaging (Vigene Bioscience, Shandong, China). The vector used was a bi-cistronic construct with EGFP. HUVECs were seeded in each well of 6-well plate $\left(5 \times 10^{3}\right.$ cells/well). Approximately $18-24 \mathrm{~h}$ later, the medium was replaced with fresh medium containing different viruses (final concentration, $2.5 \times 10^{6} \mathrm{pfu} / \mathrm{mL}$ ). At $48 \mathrm{~h}$ after initial virus treatment, the infection efficiency was evaluated using GFP fluorescence imaging (Additional file 1: Figure S3).

\section{Cell viability assay}

Infected cells $\left(5 \times 10^{3} /\right.$ well $)$ in $100 \mu \mathrm{L}$ of culture medium were seeded into 96-well plates and incubated for 18-24 h, after which the medium was replaced with a virus suspension (final concentration $2.5 \times 10^{6} \mathrm{pfu} / \mathrm{mL}$ ) in fresh medium. Afterward, the cell viability assay was performed by adding $10 \mu \mathrm{L}$ of reagent from Cell Counting Kit-8 (CCK8) (Meilunbio, cat no. MA0218-L, Dalian, China) into each well and incubating the plates for another $2 \mathrm{~h}$; then, absorbance at $450 \mathrm{~nm}$ was detected with a microplate reader (Molecular Devices, Silicon Valley, CA, USA). The cell survival rate was calculated as follows: (OD value of wild-type PIK3CD or mutant PIK3CD group/OD value of the control group) $\times 100 \%$.

\section{Quantitative real-time PCR (RT-qPCR)}

Total RNA was isolated from HUVECs using an RNA isolation kit according to the manufacturer's protocol (cat no. 220010; Shanghai feijie biological, Inc, Shanghai, China). The RNA was subsequently reverse transcribed into cDNA using a KR106-02 reverse transcription 
Table 4 Sequence of primers used for quantitative real-time PCR (RT-qPCR)

\begin{tabular}{ll}
\hline Primer & Sequence $\left(\mathbf{5}^{\prime}\right.$-3') \\
\hline GAPDH-F & 5'-GGAGCGAGATCCCTCCAAAAT -3' \\
GAPDH-R & 5'-GGCTGTTGT CATACTTCTCATGG -3' \\
mTOR-F & 5'-ATGCTTGGAACCGGACCTG -3' \\
mTOR-R & 5'-TCTTGACTCATCT CTCGGAGTT -3' (reverse) \\
AKT(Human)-F & 5'-CTACCCACACAGCAGTACGC-3' \\
AKT(Human)-R & 5'-AAGTCGCTGGTGTTAAGCCG-3' \\
S6-F & 5'-AGGGTTATGTGGTCCGAATCA -3' \\
S6-R & 5'-TTGGTCTGTAACAGGAATGCC -3' \\
\hline
\end{tabular}

Table 5 Antibodies for western blot

\begin{tabular}{llll}
\hline No & Antibodies & Company & $\begin{array}{l}\text { No. } \\
\text { product }\end{array}$ \\
\hline 1 & Anti-p-AKT antibody & Abcam & ab8933 \\
2 & Anti-AKT antibody & Abcam & ab8805 \\
3 & Anti-p-mTOR antibody & Abcam & ab109268 \\
4 & Anti-mTOR antibody & Abcam & ab32028 \\
5 & Anti-p-S6 antibody & CST & 4858 \\
6 & Anti-S6 antibody & CST & 2217 \\
\hline
\end{tabular}

system according to the manufacturer's instruction (cat. no. KR106-02; TIANGEN, Beijing, China). To detect RNA expression, qPCR analyses were carried out in triplicate using SYBR Green PCR Master Mix (cat. no. FP205-02; TIANGEN, Beijing, China) and run on a Roche LightCycler 96 (Roche Diagnostics, Indianapolis, IN, USA). Relative expression was calculated by the $2-\Delta \Delta \mathrm{Ct}$ method with GAPDH as the endogenous control. The primer sequences for the specific targets were showed in Table 4.

\section{Western blot}

Total proteins were extracted from HUVECs using RIPA buffer (cat. no, XSY-WB-001; B-Belife, Beijing China) mixed with $1 \%$ protease inhibitor cocktail (cat. no. 04693116001; Roche Molecular Biochemicals, Mannheim, Germany). The protein concentration was determined using a BCA Protein assay kit (cat. no. CW0014S; CWBIO, Beijing, China). Protein extracts were then mixed with $5 \times$ SDS loading buffer and boiled for $10 \mathrm{~min}$. Then $20-50 \mu \mathrm{g}$ per sample were separated via SDS-PAGE under reducing or non-reducing conditions on a $10 \%$ polyacrylamide gel and then electrotransferred onto PVDF membranes (Millipore, Bedford, MA, USA) in vertical buffer tanks. The membranes were blocked with $5 \%$ non-fat milk in TBST buffer $(50 \mathrm{mM}$ Tris- $\mathrm{HCl}(\mathrm{pH}$
7.4), $0.9 \% \mathrm{NaCl}$, and $0.1 \%$ Tween 20 ) before they were incubated with primary antibodies (Table 5) for $2-3 \mathrm{~h}$ at room temperature or overnight at $4{ }^{\circ} \mathrm{C}$. After the addition of the HRP-conjugated secondary antibodies for $1 \mathrm{~h}$, signals were detected with an electrogenerated chemiluminescence (ECL) detection reagent (cat. no. MA0186; Meilunbio, Dalian, China). Relative target protein expression levels were normalized to those of tubulin and visualized using ImageJ software.

\section{Wound healing assay}

The migration ability of HUVECs were assessed by the wound healing assay. A sterile tip was used to create a wound in a cell monolayer, which was then washed 3 times to remove non-adherent cells, and fresh medium was added to the cultures. Images were captured at $0,4,8$ and $12 \mathrm{~h}$ after scratching. Photoshop software (Adobe Photoshop CS6) was used to measure the area of the wound at each time point and calculate the wound healing rate as follows: migration area $(\%)=\left(\mathrm{A}_{0}-\mathrm{A}_{\mathrm{n}}\right) /$ $A_{0} \times 100$, where $A_{0}$ represents the area of initial wound area, an represents the remaining area of wound at the metering time point.

\section{Statistical analysis}

All in vitro experiments performed in this study were repeated three times. Statistical analysis was performed using GraphPad Prism software, and all comparisons between groups were assessed using Student's t test. All clinical data are indicated as mean \pm standard deviation (Mean \pm SD), with the significant statistical threshold of two-tailed $p$ value $<0.05$.

\section{Supplementary Information}

The online version contains supplementary material available at https://doi. org/10.1186/s13023-021-01782-9.

Additional file 1: Figure S1. Pathological structure of each subtype of LM. Figure S2. Schematic diagram of the bioinformatics pipeline to analyze the targeted deep sequencing data. Figure $\mathbf{S 3}$. Transfection rate quantified by qPCR. Table S1: The coverage of each sample and average depth.

\section{Acknowledgements}

We are grateful to the children with lymphatic malformations and their families who have willingly participated in this study.

\section{Authors' contributions}

Conception and design: X.N., J.T., W.S. and W.W.; patient data and samples: W.S., X.Z., J.Z., Q.L., Y.L. and N.S.; experiments, collection and assembly of data:W.W., X.Z., Y.G., Z.L. and J.Z.; data analysis and interpretation:W.S., W.W., L.H. and J.G.; manuscript writing and editing: W.S., W.W., X.Z., L.H. and J.Z.

\section{Funding}

This research was supported by Natural Science Foundation of Beijing Municipality (7182050); Beijing Municipal Administration of Hospitals Clinical 
Technology Innovation Project Grant (XMLX201701). Beijing Hospitals Authority Youth Programme (QML20181202).

\section{Availability of data and materials}

The datasets used and analysed during the current study are available from the corresponding author on reasonable request.

\section{Declarations}

\section{Ethics approval and consent to participate}

Guardians of all the patients signed informed consent in accordance with the Declaration of Helsinki. LM specimens were obtained under the human subject protocol approved by the Human Ethics Committee of Beijing Children's Hospital Affiliated to Capital Medical University (2019-k-66, approved on February 2019).

\section{Consent for publication}

Guardians of all the patients agree with the publication of the manuscript.

\section{Competing interests}

The authors declare that they have no competing interests.

\begin{abstract}
Author details
'Department of Otolaryngology-Head and Neck Surgery, Beijing Children's Hospital, Capital Medical University, National Center for Children's Health, Beijing 100045, China. ${ }^{2}$ Laboratory of Tumor Immunology, Beijing Pediatric Research Institute, Beijing Children's Hospital, Capital Medical University, National Center for Children's Health, Beijing 100045, China. ${ }^{3}$ Beijing Key Laboratory for Pediatric Diseases of Otolaryngology, Head and Neck Surgery, Beijing Pediatric Research Institute, Beijing Children's Hospital, Capital Medical University, National Center for Children's Health, Beijing 100045, China. ${ }^{4}$ Running-Gene Inc., Health Valley 602, Beijing, China.
\end{abstract}

Received: 4 August 2020 Accepted: 16 March 2021

Published online: 08 May 2021

\section{References}

1. Kennedy TL, Whitaker M, Pellitteri P, Wood WE. Cystic hygroma/lymphangioma: a rational approach to management. Laryngoscope. 2001;111(11 Pt 1):1929-37.

2. Elluru RG, Balakrishnan K, Padua HM. Lymphatic malformations: diagnosis and management. Semin Pediatr Surg. 2014;23(4):178-85.

3. Heit JJ, Do HM, Prestigiacomo CJ, Delgado-Almandoz JA, English J, Gandhi CD, Albuquerque FC, Narayanan S, Blackham KA, Abruzzo T, Albani B, Fraser JF, Heck DV, Hussain MS, Lee SK, Ansari SA, Hetts SW, Bulsara KR, Kelly M, Arthur AS, Patsalides A, Pride GL, Powers CJ, Alexander MJ, Meyers PM, Jayaraman MV. Guidelines and parameters: percutaneous sclerotherapy for the treatment of head and neck venous and lymphatic malformations. J Neurointerv Surg. 2017;9(6):611-7.

4. Wiegand S, Wichmann G, Dietz A. Treatment of lymphatic malformations with the mTOR inhibitor sirolimus: a systematic review. Lymphat Res Biol. 2018;16(4):330-9.

5. Bagrodia N, Defnet AM, Kandel JJ. Management of lymphatic malformations in children. Curr Opin Pediatr. 2015;27(3):356-63.

6. Yadav P, De Castro DK, Waner M, Meyer L, Fay A. Vascular anomalies of the head and neck: a review of genetics. Semin Ophthalmol. 2013;28(5-6):257-66.

7. Boscolo E, Coma S, Luks VL, Greene AK, Klagsbrun M, Warman ML, Bischoff J. AKT hyper-phosphorylation associated with PI3K mutations in lymphatic endothelial cells from a patient with lymphatic malformation. Angiogenesis. 2015;18(2):151-62.

8. Luks VL, Kamitaki N, Vivero MP, Uller W, Rab R, Bovee JV, Rialon KL, Guevara CJ, Alomari Al, Greene AK, Fishman SJ, Kozakewich HP, Maclellan RA, Mulliken JB, Rahbar R, Spencer SA, Trenor CC, Upton J, Zurakowski D, Perkins JA, Kirsh A, Bennett JT, Dobyns WB, Kurek KC, Warman ML, McCarroll SA, Murillo R. Lymphatic and other vascular malformative/overgrowth disorders are caused by somatic mutations in PIK3CA. J Pediatr. 2015;166(4):1048-54.
9. Brouillard P, Boon L, Vikkula M. Genetics of lymphatic anomalies. J Clin Invest. 2014;124(3):898-904.

10. Lindhurst MJ, Sapp JC, Teer JK, Johnston JJ, Finn EM, Peters K, Turner J, Cannons JL, Bick D, Blakemore L, Blumhorst C, Brockmann K, Calder P, Cherman N, Deardorff MA, Everman DB, Golas G, Greenstein RM, Kato BM, Keppler-Noreuil KM, Kuznetsov SA, Miyamoto RT, Newman K, Ng D, O'Brien K, Rothenberg S, Schwartzentruber DJ, Singhal V, Tirabosco R, Upton J, Wientroub S, Zackai EH, Hoag K, Whitewood-Neal T, Robey PG, Schwartzberg PL, Darling TN, Tosi LL, Mullikin JC, Biesecker LG. A mosaic activating mutation in AKT1 associated with the Proteus syndrome. N Engl J Med. 2011;365(7):611-9.

11. Zenner K, Cheng CV, Jensen DM, et al. Genotype correlates with clinical severity in PIK3CA-associated lymphatic malformations. JCI Insight. 2019:4(21):e129884.

12. Blesinger $H$, Kaulfuss $S$, Aung T, et al. PIK3CA mutations are specifically localized to lymphatic endothelial cells of lymphatic malformations. PLoS ONE. 2018;13(7):e0200343.

13. Osborn AJ, Dickie P, Neilson DE, Glaser K, Lynch KA, Gupta A, Dickie BH. Activating PIK3CA alleles and lymphangiogenic phenotype of lymphatic endothelial cells isolated from lymphatic malformations. Hum Mol Genet. 2015;24(4):926-38.

14. Li MM, Datto M, Duncavage EJ, Kulkarni S, Lindeman NI, Roy S, Tsimberidou AM, Vnencak-Jones CL, Wolff DJ, Younes A, Nikiforova MN. Standards and guidelines for the interpretation and reporting of sequence variants in cancer: a joint consensus recommendation of the association for molecular pathology, American Society of Clinical Oncology, and College of American Pathologists. J Mol Diagn. 2017;19(1):4-23.

15. Okkenhaug K. Signaling by the phosphoinositide 3 -kinase family in immune cells. Annu Rev Immunol. 2013;31:675-704.

16. Okkenhaug K, Vanhaesebroeck B. PI3K in lymphocyte development, differentiation and activation. Nat Rev Immunol. 2003:3(4):317-30.

17. Fruman DA, Chiu H, Hopkins BD, Bagrodia S, Cantley LC, Abraham RT. The PI3K pathway in human disease. Cell. 2017;170(4):605-35.

18. Thorpe LM, Yuzugullu H, Zhao JJ. PI3K in cancer: divergent roles of isoforms, modes of activation and therapeutic targeting. Nat Rev Cancer. 2015;15(1):7-24.

19. Fruman DA, Rommel C. PI3K and cancer: lessons, challenges and opportunities. Nat Rev Drug Discov. 2014;13(2):140-56.

20. Wojtalla A, Fischer B, Kotelevets N, Mauri FA, Sobek J, Rehrauer H, Wotzkow C, Tschan MP, Seckl MJ, Zangemeister-Wittke U, Arcaro A. Targeting the phosphoinositide 3-kinase p110-alpha isoform impairs cell proliferation, survival, and tumor growth in small cell lung cancer. Clin Cancer Res. 2013;19(1):96-105.

21. An HJ, Cho NH, Yang HS, Kwak KB, Kim NK, Oh DY, Lee SW, Kim HO, Koh J. Targeted RNA interference of phosphatidylinositol 3-kinase p110-beta induces apoptosis and proliferation arrest in endometrial carcinoma cells. J Pathol. 2007:212(2):161-9.

22. Limaye N, Kangas J, Mendola A, Godfraind C, Schlogel MJ, Helaers R, Eklund L, Boon LM, Vikkula M. Somatic Activating PIK3CA Mutations Cause Venous Malformation. Am J Hum Genet. 2015;97(6):914-21.

23. Luk SK, Piekorz RP, Nurnberg B, Tony To SS. The catalytic phosphoinositol 3-kinase isoform p1 10delta is required for glioma cell migration and invasion. Eur J Cancer. 2012;48(1):149-57.

24. Eickholt BJ, Ahmed Al, Davies M, Papakonstanti EA, Pearce W, Starkey ML, Bilancio A, Need AC, Smith AJ, Hall SM, Hamers FP, Giese KP, Bradbury EJ, Vanhaesebroeck B. Control of axonal growth and regeneration of sensory neurons by the p110delta PI 3-kinase. PLoS ONE. 2007;2(9):e869.

25. Puri KD, Doggett TA, Douangpanya J, Hou Y, Tino WT, Wilson T, GrafT, Clayton E, Turner M, Hayflick JS, Diacovo TG. Mechanisms and implications of phosphoinositide 3-kinase delta in promoting neutrophil trafficking into inflamed tissue. Blood. 2004;103(9):3448-56.

26. Conte E, Fruciano M, Fagone E, Gili E, Caraci F, lemmolo M, Crimi N, Vancheri C. Inhibition of PI3K prevents the proliferation and differentiation of human lung fibroblasts into myofibroblasts: the role of class I P110 isoforms. PLoS ONE. 2011;6(10):e24663.

27. Shi Y, Chen C, Zhang X, Liu Q, Xu JL, Zhang HR, Yao XH, Jiang T, He ZC, Ren Y, Cui W, Xu C, Liu L, Cui YH, Yu SZ, Ping YF, Bian XW. Primatespecific miR-663 functions as a tumor suppressor by targeting PIK3CD and predicts the prognosis of human glioblastoma. Clin Cancer Res. 2014;20(7):1803-13. 
28. Boller D, Schramm A, Shalaby DKT, von Bueren AO, Eggert A, Grotzer MA, Arcaro A. Targeting the phosphoinositide 3-kinase isoform p110delta impairs growth and survival in neuroblastoma cells. Clin Cancer Res. 2008;14(4):1172-81.

29. Sawyer C, Sturge J, Bennett DC, O'Hare MJ, Allen WE, Bain J, Jones GE, Vanhaesebroeck B. Regulation of breast cancer cell chemotaxis by the phosphoinositide 3-kinase p1 10delta. Cancer Res. 2003:63(7):1667-75.

30. Chen JS, Huang JQ, Luo B, Dong SH, Wang RC, Jiang ZK, Xie YK, Yi W, Wen GM, Zhong JF. PIK3CD induces cell growth and invasion by activating AKT/GSK-3beta/beta-catenin signaling in colorectal cancer. Cancer Sci. 2019;110(3):997-1011.

31. Wang Q, Wang J, Wang M, Xu Y, Xu MN, Yuan SM. The mTOR signal pathway is overactivated in human lymphatic malformations. Lymphat Res Biol. 2019;17(6):624-9.

32. Hammer J, Seront E, Duez S, Dupont S, Van Damme A, Schmitz S, Hoyoux C, Chopinet C, Clapuyt P, Hammer F, Vikkula M, Boon LM. Sirolimus is efficacious in treatment for extensive and/or complex slow-flow vascular malformations: a monocentric prospective phase II study. Orphanet J Rare Dis. 2018;13(1):191.

33. Xuexi Zhang: Shengcai Wang: Yongli Guo; Yuanhu Liu; Jie Zhang:Yanzhen Li; Qiaoyin Liu; Zhiyong Liu; Nian Sun; Xiaodan Li; Yuwei Liu; Jiangnan Du;
Xiaoling Cheng; Xiaoling Wang; Jun Tai; Xin Ni. Efficacy of initial sirolimus therapy for 27 patients with intractable lymphatic malformations. The Laryngoscope. 2021 (Accepted).

34. Martinez-Corral I, Zhang Y, Petkova M, et al. Blockade of VEGF-C signaling inhibits lymphatic malformations driven by oncogenic PIK3CA mutation. Nat Commun. 2020;11(1):2869.

35. Scotting PJ, Walker DA, Perilongo G. Childhood solid tumours: a developmental disorder. Nat Rev Cancer. 2005;5(6):481-8.

36. Venot $\mathrm{Q}$, Blanc T, Rabia SH, Berteloot L, Ladraa S, Duong JP, Blanc E, Johnson SC, Hoguin C, Boccara O, Sarnacki S, Boddaert N, Pannier S, Martinez F, Magassa S, Yamaguchi J, Knebelmann B, Merville P, Grenier N, Joly D, Cormier-Daire V, Michot C, Bole-Feysot C, Picard A, Soupre V, Lyonnet S, Sadoine J, Slimani L, Chaussain C, Laroche-Raynaud C, Guibaud L, Broissand C, Amiel J, Legendre C, Terzi F, Canaud G. Targeted therapy in patients with PIK3CA-related overgrowth syndrome. Nature. 2018;558(7711):540-6.

\section{Publisher's Note}

Springer Nature remains neutral with regard to jurisdictional claims in published maps and institutional affiliations.
Ready to submit your research? Choose BMC and benefit from:

- fast, convenient online submission

- thorough peer review by experienced researchers in your field

- rapid publication on acceptance

- support for research data, including large and complex data types

- gold Open Access which fosters wider collaboration and increased citations

- maximum visibility for your research: over $100 \mathrm{M}$ website views per year

At BMC, research is always in progress.

Learn more biomedcentral.com/submissions 